\title{
AVALIAÇÃO DE MÉTODO DE QUESTIONÁRIO PARA ESTUDO DA RELAÇÃO ENTRE PRESENÇA E USO DE APARELHOS DE REFRIGERAÇÃO, ARBORIZAÇÃO VIÁRIA E CONSUMO DE ENERGIA ELÉTRICA ${ }^{1}$
}

\author{
Giuliana Del Nero Velasco², Ana Maria Liner Pereira Lima³, Hilton Thadeu Zarate do Couto4, \\ Demóstenes Ferreira da Silva Filho ${ }^{4}$ e Jefferson Lordello Polizel ${ }^{4}$
}

\begin{abstract}
RESUMO - Dentre os inúmeros benefícios da arborização viária, destaca-se a capacidade de redução da temperatura do ar, seja pelo sombreamento causado pela copa das árvores, seja pela evapotranspiração. O aumento de temperatura em áreas urbanas eleva a demanda pelo resfriamento do ar, afetando dramaticamente os custos de energia elétrica. O objetivo deste trabalho foi o de avaliar a aplicação de questionários para o estudo da relação entre a vegetação viária na cidade de São Paulo, o consumo de energia elétrica e o uso de aparelhos de refrigeração em residências. Foram escolhidas três áreas na cidade de São Paulo/SP que diferissem em termos de quantidade de vegetação, selecionadas através de amostragem sistemática e 100 residências em cada área, nas quais foram aplicados questionários e analisadas as contas de energia elétrica. Conclui-se que o uso do questionário não foi eficiente para estabelecer uma relação entre a vegetação viária, o consumo de energia elétrica em residências e o uso de aparelhos de refrigeração. Isso porque não houve diferença estatística tanto entre os hábitos de uso de aparelhos de ar condicionado, quanto na quantidade de tais aparelhos nas três áreas. Ao contrário, o consumo de energia elétrica diferiu entre as áreas, ou seja, apenas com os dados coletados nos questionários não foi possível isolar o efeito dos aparelhos de ar condicionado no valor total do consumo de energia elétrica, já que tal consumo variou nas três áreas, mas a quantidade e o uso não diferiram
\end{abstract}

Palavras-chave: Questionários, Arborização viária e Energia elétrica.

\section{ASSESSMENT OF QUESTIONNAIRES METHOD TO STUDY OF THE RELATIONSHIP BETWEEN THE PRESENCE AND USE OF REFRIGERATION APPLIANCES, STREET TREES AND ELECTRIC POWER CONSUMPTION}

\begin{abstract}
Among of the many benefits of street trees, it stands out the capacity to reduce air temperature, either by shading caused by tree crowns, or by evapotranspiration. The increase of temperature in urban areas raises the demand for air cooling, affecting drastically the cost of electric energy. The objective of this study was to evaluate the application of questionnaires to study the relationship between the presence of street trees in the city of São Paulo, the electric power consumption and the presence and use of air conditioning appliances in the residences. It was chosen three areas in São Paulo which differed in the quantity of vegetation, selected by systematic sampling and 100 residences in each area, where the questionnaires were applied and electric power bills were analyzed. The use of questionnaire was not sufficient to establish a relationship between a relationship between the presence of roadside vegetation, electric power consumption in residences and the presence and use of refrigeration appliances. This is because there was no statistical difference among habits of air conditioning use as well as on the quantity of such appliances in the three areas. On the contraty, electric energy consumption differed among areas, that is, only with data collected from the questionnaires was not possible to isolate the effect of air conditioning appliances in the total value of electric energy consumption inasmuch as such consumption varied in the three areas, but quantity and and use did not differ.
\end{abstract}

Keywords: Electric energy, Questionnaires and Street trees.

\footnotetext{
${ }^{1}$ Recebido em 18.08.2009 e aceito para publicação em 18.04.2011.

${ }^{2}$ Doutora em Agronomia. E-mail: <giulivelasco@hotmail.com>.

${ }^{3}$ Departamento de Produção Vegetal, ESALQ- USP, Brasil. E-mail: <amlplima@esalq.usp.br>.

${ }^{4}$ Departamento de Ciências Florestais, ESALQ/USP. Email: <htzcouto@esalq.usp.br>, <dfilho@usp.br>, <jlpolize@esalq.usp.br>
} 


\section{INTRODUÇÃO}

Um ambiente urbano é caracterizado pela interação de elementos naturais e construídos, bem como por uma dinâmica de ocupação do solo por construções, veículos, pedestres, entre outros, os quais resultam em uma alteração no clima local. Segundo Keeble, et al. (1990/91), o aumento de áreas construídas leva a uma degradação ambiental, seja através da poluição gerada, seja pelo aumento do estresse causado pelo aquecimento, principalmente em locais de temperatura média mais alta.

Em um ambiente urbano, o concreto, pedra, tijolo e asfalto substituem a cobertura vegetal, resultando em conseqüências negativas como: intensificação dos piores aspectos do clima urbano; demanda crescente de energia; redução da absorção dos poluentes atmosféricos; enchentes cada vez maiores; degradação da qualidade das águas e, em áreas residenciais, depreciação das propriedades (SPIRN, 1995).

As elevadas temperaturas aumentam os níveis de poluição e a necessidade de uso de aparelhos de refrigeração(SCHILLER; EVANS, 1996), bem como podem modificar o regime de chuvas da região (YUAN; BAUER, 2007). Para Wilmers (1988), os dois principais aspectos negativos do clima urbano são as ilhas de calor e a poluição do ar, os quais podem ser amenizados com a presença de vegetação. A sombra das árvores e as superfícies com alto albedo devem ser usadas como estratégias de baixo custo na redução das ilhas de calor urbanas (BRETZ; AKBARI; ROSENFELD, 1998; ROSENFELD, et al., 1998).

Ainda em termos de estresse térmico, Santamouris (2001) afirma que o aumento de temperatura em áreas urbanas eleva a demanda pelo resfriamento do ar, afetando dramaticamente os custos de energia elétrica, como visto em pesquisa realizada no exterior (Estados Unidos, 2007). O resultado de tal pesquisa apontou um aumento de $1,5-2,0 \%$ na demanda de resfriamento a cada $0,6^{\circ} \mathrm{C}$ de aumento de temperatura.

A obtenção de energia pode ser vista, atualmente, como uma fonte de problemas ambientais, seja para produção de eletricidade, setor de transportes ou indústrias. Em termos de energia elétrica, pode-se dizer que este setor é responsável por aproximadamente um terço do consumo de energia primária mundial e as tendências indicam que essa contribuição vai aumentar nas próximas décadas, já que ainda tem-se uma realidade de dois bilhões de pessoas sem acesso à eletricidade (GOLDEMBERG; VILLANUEVA, 2003).

A dificuldade de desenvolver pesquisas sobre uso e economia de energia elétrica no setor residencial é grande, visto ser fortemente influenciada pelas atitudes e comportamentos de seus moradores. Cavalcanti (2002) afirma que o uso de energia na classe residencial está ligado ao hábito de consumo e posse de eletrodomésticos, o que gera uma grande limitação devido às inúmeras variáveis que influenciam o consumo de energia na classe residencial (tanto técnicas como comportamentais). A autora afirma a necessidade de estudar os hábitos de consumo e posse de eletrodomésticos no setor residencial, de modo a se definir o perfil deste consumidor e, por conseguinte, implementar programas de conservação dessa energia.

O uso de questionários é uma ferramenta utilizada em pesquisas com arborização urbana, devendo ser testada no estudo da relação entre arborização viária e o consumo de energia elétrica. Em arborização urbana alguns trabalhos já foram desenvolvidos com o uso de questionários como Porfírio et al. (2006) ao analisar a interação de moradores de três bairros com o Parque Municipal das Mangabeiras em Belo Horizonte (MG) e Hildebrand et al. (2001) ao utilizarem o questionário para estudo de parques urbanos em Curitiba (PR).

O objetivo do presente trabalho foi o de avaliar a aplicação de questionários para o estudo da relação entre a presença de vegetação viária na cidade de São Paulo e o consumo de energia elétrica com a presença e uso de aparelhos de refrigeração em residências.

\section{MATERIAL E MÉTODOS}

Foram escolhidas três áreas para o presente estudo com baixa, média e alta densidade de vegetação. Tal escolha foi feita com base na pesquisa de Velasco et al. (2007) que testaram a aplicação do índice de vegetação, a partir de imagens de satélite de alta resolução. Com o resultado deste estudo foi possível escolher três áreas distintas em termos de densidade de vegetação. O uso de ferramentas do sensoriamento remoto para caracterização da cobertura vegetal em cidades é uma prática muito utilizada, principalmente, em áreas muito extensas. Assim, é possível caracterizar uma região ou mesmo uma cidade em suas diferentes coberturas

Revista Árvore, Viçosa-MG, v.35, n.3, Edição Especial, p.641-648, 2011 
(asfalto, vegetação, solo nu, cursos d'água, entre outros). Vieira e Biondi (2008) também utilizaram o sensoriamento remoto para analisar a cobertura vegetal da cidade de Curitiba, ao longo dos anos, com o uso de imagens do satélite Landsat TM.

Dentro de cada uma das três áreas de estudo foram selecionados cem pontos (cem residências), definidas através de amostragem sistemática, que permitiu uma distribuição uniforme das amostras, representando adequadamente a área de estudo, com erro máximo de $10 \%$ e com $95 \%$ de probabilidade.

Desta forma, foram selecionadas as ruas que estão dentro de cada área e cada rua foi divida em quarteirões; dividiu-se o número de cem residências pelo total de quarteirões, resultando no número de residências a serem amostradas por quarteirão.

Foi elaborado um questionário (Figura 1) que foi aplicado nas trezentas residências amostradas, no período de janeiro a março de 2007. Buscou-se levantar a quantidade dos principais elementos que interferiam no consumo de energia elétrica bem como o hábito de uso dos aparelhos de refrigeração. Foi feita uma análise, comparando as três áreas, verificando se tais parâmetros diferiam ou não entre áreas. Caso a quantidade de aparelhos de ar condicionado e seu uso fossem diferentes entre áreas, seria analisada sua contribuição no consumo final de cada residência.

Foi feita a análise de variância e a aplicação do teste Tukey, a 5\% de significância, bem como a análise de tabela de contingência, usando-se o teste G (razão da máxima verossimilhança). As análises foram feitas utilizando-se o programa estatístico SAS (Statistical Analysis System).

Foram obtidos, junto à concessionária de energia elétrica, AES ELETROPAULO, dados de consumo (em kWh - quilowatt-hora) das trezentas residências amostradas, dos meses de Setembro de 2006 e Março de 2007. Foi feita uma média de consumo de energia elétrica, por área, das residências que responderem os questionários.

\section{RESULTADOS}

As três áreas escolhidas estão localizadas na zona Sul da cidade de São Paulo, uma com baixa, uma com média e uma com alta densidade de vegetação.
Das trezentas residências, cento e vinte questionários foram obtidos. Os principais motivos da não participação dos moradores foram a falta de tempo, o desinteresse pelo assunto e medo.

Da análise dos resultados obtidos nos questionários, pode-se dizer que a quantidade de residências que possuía aparelhos de refrigeração era baixa (apenas vinte e uma residências dos cento e vinte questionários respondidos).

O consumo médio de energia elétrica por residência foi calculado utilizando as 120 residências que participaram da pesquisa, respondendo o questionário.

A Tabela 1 mostra o resultado da análise feita com uso do programa estatístico SAS, a partir dos dados obtidos dos questionários, nas três áreas, indicando que não houve diferença estatística, entre áreas, quanto à quantidade de aparelhos de ar condicionado, ao contrário do consumo médio entre as três áreas, que diferiu estatisticamente, como pode ser visto na Tabela 2.

O aparelho que mais diferiu, em quantidade, entre as áreas 1 e 3, foi o freezer, mas provavelmente a diferença entre consumo nas áreas não se deu apenas devido à quantidade diferente de freezers. Segundo dados do PROCEL, 2005 apud Francisco (2006) um freezer de 1300 Watts de potência consome mensalmente, em média, 50kWh, não podendo ser responsabilizado pela diferença significativa de consumo entre áreas.

Analisando separadamente cada uma das áreas e caracterizando as residências quanto à quantidade de aparelhos de ar condicionado, tem-se na Tabela 3, o consumo médio nas áreas 1, 2 e 3.

Pode-se afirmar que, dentro dos valores estudados, o consumo médio foi, na maioria das situações, positivamente relacionado à variável ar condicionado, ou seja, as casas que não continham tal eletrodoméstico tinham valores de consumo menores quando comparadas às casas com três aparelhos.

Em relação ao uso dos aparelhos de ar condicionado, apresenta-se na Tabela 4 o resultado da análise estatística, observando-se que os valores não diferiram estatisticamente $(P>0,05)$, não havendo diferença entre os hábitos de uso de aparelhos de ar condicionado, nas três áreas. 
1. Número de pessoas na casa: Moradores ( ) Funcionários ( )

Quantas ficam mais tempo em casa de manhã ( ) a tarde ( ) a noite ( ) dia todo ( )

2. Qual o total de área construída? Quantos quartos ( ), salas ( ), banheiros ( )

3. Tem árvore(s) dentro do terreno? ( ) Sim ()Não Quantas ( ) Tamanho:

4. O sol da manhã bate em quais cômodos da casa? ( ) quartos ( ) salas ( ) cozinha ( ) outros

5. O sol da tarde bate em quais cômodos da casa? () quartos () salas ( ) cozinha ( ) outros

6. Quantos desses aparelhos têm na sua residência?

Chuveiro elétrico ( ) Geladeira () Freezer ( ) Aparelho de Som ( )T.V( ) DVD ( ) Computador ( ) Microondas ( )

vídeo cassete ( ) Torneira elétrica ( ) luminárias de jardim (spots) ( ) Máquina de lavar roupa ( ) Máquina de lavar louça ( ) Ferro de passar roupa ( )

7. Qual é o tipo de aquecimento (água)? Elétrico ( ) Solar ( ) A gás ( )

8. Em quais dos três meses: dezembro ( ) janeiro ( ) ou fevereiro ( ) que mais tem gente em casa?

9. Quantos aparelhos de refrigeração têm na sua casa? Ar condicionado（） Ventilador（）

10. Em quais cômodos eles se encontram?

Ar condicionado: ( ) quartos ( ) salas ( ) cozinha ( ) outros

Ventilador: ( ) quartos ( ) salas ( ) cozinha ( ) outros

11. Como usa cada um dos aparelhos? Colocar entre parênteses o número de aparelhos referente a cada forma de uso. Ar condicionado:

( ) Ligo apenas quando está muito calor. Qual horário?

( ) deixo sempre ligado. Qual horário?

( ) Ligo apenas durante o dia (em todas as épocas do ano). Qual horário?

( ) Ligo apenas durante o dia (nos dias quentes). Qual horário?

( ) Ligo apenas durante a noite (em todas as épocas do ano). Qual horário?

( ) Ligo apenas durante a noite (nos dias quentes). Qual horário?

Ventilador

( ) Ligo apenas quando está muito calor. Qual horário?

() deixo sempre ligado. Qual horário?

( ) Ligo apenas durante o dia (em todas as épocas do ano). Qual horário?

( ) Ligo apenas durante o dia (nos dias quentes). Qual horário?

( ) Ligo apenas durante a noite (em todas as épocas do ano). Qual horário?

( ) Ligo apenas durante a noite (nos dias quentes). Qual horário?

12. Em quais dos três meses: junho ( ) julho ( ) ou agosto ( ) que mais tem gente em casa?

13. Quantos aquecedores de ar têm na sua casa?

14. Em quais cômodos eles se encontram?

15. Como você usa esses aparelhos? Colocar entre parênteses o número de aparelhos referente a cada forma de uso.

( ) Ligo apenas quando está muito frio. Qual horário

() deixo sempre ligado. Qual horário?

( ) Ligo apenas durante o dia (em todas as épocas do ano). Qual horário?

( ) Ligo apenas durante o dia (nos dias frios). Qual horário?

( ) Ligo apenas durante a noite (em todas as épocas do ano). Qual horário?

( ) Ligo apenas durante a noite (nos dias frios). Qual horário?

Figura 1 - Questionário aplicado às residências, São Paulo, 2007.

Figure 1-Questionnaire applied to the residences, São Paulo, 2007. 
Tabela 1 - Médias por residência dos dados obtidos na aplicação dos questionários, nas três áreas, São Paulo/SP, 2007. Table 1 - Means of of each residence in the data obtained by applying questionnaires in the three areas, São Paulo/SP, 2007.

\begin{tabular}{|c|c|c|c|}
\hline & $\begin{array}{c}\text { Área } 1 \text { (baixa } \\
\text { vegetação) }\end{array}$ & $\begin{array}{l}\text { Área } 2 \text { (média } \\
\text { vegetação) }\end{array}$ & $\begin{array}{r}\text { Área } 3 \text { (alta } \\
\text { vegetação) }\end{array}$ \\
\hline Quantidade de moradores & $3,37 a$ & $3,58 a$ & $3,38 a$ \\
\hline Quantidade de funcionários & $0,63 b$ & $1,07 a$ & $1,23 a$ \\
\hline Permanência no período da manhã & $1,08 a b$ & $0,87 b$ & $1,50 \mathrm{a}$ \\
\hline Permanência no período da tarde & $0,63 b$ & $0,91 \mathrm{ab}$ & $1,47 \mathrm{a}$ \\
\hline Permanência no período da noite & $2,58 a$ & $3,04 a$ & $3,38 a$ \\
\hline Permanência durante o dia todo & $1,34 \mathrm{a}$ & $1,38 \mathrm{a}$ & $1,82 \mathrm{a}$ \\
\hline Área construída $\left(\mathrm{m}^{2}\right)$ & $206,00 b$ & $256,48 b$ & $381,50 a$ \\
\hline Quantidade de quartos & $3,13 a$ & $3,43 a$ & $3,53 a$ \\
\hline Quantidade de salas & $1,74 b$ & $2,30 \mathrm{a}$ & $1,85 b$ \\
\hline Quantidade de banheiros & $3,26 a$ & $3,85 a$ & $3,76 a$ \\
\hline Quantidade de chuveiro elétrico & $2,5 a$ & $1,84 \mathrm{~b}$ & $2,47 a$ \\
\hline Quantidade de freezer & $0,58 b$ & $0,51 b$ & $1,03 a$ \\
\hline Quantidade de geladeira & $1,24 a$ & $1,51 \mathrm{a}$ & $1,44 \mathrm{a}$ \\
\hline Quantidade de aparelho de som & $1,55 a$ & $1,69 a$ & $1,94 a$ \\
\hline Quantidade de aparelho de Televisão & $2,63 a$ & $2,69 a$ & $2,62 \mathrm{a}$ \\
\hline Quantidade de aparelho de DVD & $1,26 a$ & $1,27 a$ & $1,32 \mathrm{a}$ \\
\hline Quantidade de computador & $1,13 a$ & $1,31 \mathrm{a}$ & $1,41 \mathrm{a}$ \\
\hline Quantidade de microondas & $0,87 a$ & $0,93 a$ & $1,00 \mathrm{a}$ \\
\hline Quantidade de videocassete & $0,79 a$ & $0,56 a$ & $0,55 a$ \\
\hline Quantidade de torradeira elétrica & $0,71 \mathrm{a}$ & $0,44 a$ & $0,56 a$ \\
\hline Quantidade de luminárias de jardim (spot) & $1,13 b$ & $1,75 \mathrm{ab}$ & $2,70 \mathrm{a}$ \\
\hline Quantidade de máquina de lavar roupa & $1,05 \mathrm{a}$ & $1,00 \mathrm{a}$ & $0,94 a$ \\
\hline Quantidade de máquina de lavar louça & $0,42 \mathrm{a}$ & $0,51 \mathrm{a}$ & $0,38 a$ \\
\hline Quantidade de ferro de passar roupa & $1,21 \mathrm{a}$ & $1,13 a$ & $1,08 \mathrm{a}$ \\
\hline Quantidade de ar condicionado & $0,18 a$ & $0,24 a$ & $0,29 a$ \\
\hline Quantidade de ventiladores & $1,81 \mathrm{a}$ & $2,04 \mathrm{a}$ & $1,68 \mathrm{a}$ \\
\hline Quantidade de aquecedor de ar & $0,05 a$ & $0,19 a$ & $0,00 \mathrm{a}$ \\
\hline
\end{tabular}

Nota: Médias seguidas de letras diferentes, na linha, diferem $(\mathrm{P}<0,05)$ pelo teste Tukey.

Tabela 2 - Consumo médio por residência (kWh), nos meses de setembro de 2006 e março de 2007, nas três áreas, São Paulo/SP.

Table 2 - Consumption per residence ( $k$ Wh), from September 2006 to March 2007, in the three areas, São Paulo/SP.

\begin{tabular}{lccc}
\hline Consumo médio por residência $(\mathrm{kWh})$ & $\begin{array}{c}\text { Área 1 (baixa } \\
\text { vegetação) }\end{array}$ & $\begin{array}{c}\text { Área 2 (média } \\
\text { vegetação) }\end{array}$ & $\begin{array}{c}\text { Área 3 (alta } \\
\text { vegetação) }\end{array}$ \\
\hline setembro 2006 & $351.32 \mathrm{c}$ & $521.06 \mathrm{~b}$ & $695.21 \mathrm{a}$ \\
março 2007 & $352.45 \mathrm{c}$ & $480.61 \mathrm{~b}$ & $656.24 \mathrm{a}$ \\
\hline
\end{tabular}

Nota: Médias seguidas de letras diferentes, na linha, diferem $(\mathrm{P}<0,05)$ pelo teste Tukey.

Na Figura 2 apresenta-se a relação entre a área construída (em m²) e o consumo de energia (em kWh) indicando que as áreas 1 e 2 não tiveram resultados significativos. Aárea 3, tanto no mês de setembro quanto em março, teve resultados significantes ao nível de 0,05 apresentando uma relação de crescimento exponencial entre consumo e área construída. Francisco (2006), em sua dissertação de mestrado, ao estudar a relação existente entre o consumo de energia elétrica, a renda e a caracterização econômica de famílias de baixa renda no município de São Paulo, obteve resultados considerados significantes ao nível de 0,01, chegando a valores de $\mathrm{R}^{2}$ de 0,402. 
Tabela 3 - Consumo médio (kWh), por área, das residências com 0, 1, 2 e 3 aparelhos de ar condicionado nos meses de setembro de 2006 e março de 2007, São Paulo/SP.

Table 3-Average consumption ( $\mathrm{kWh}$, per area of residences with 0, 1, 2 and 3 air conditionings, for the months of September 2006 and March 2007, Sao Paulo/SP.

\begin{tabular}{|c|c|c|c|c|c|}
\hline & \multirow[t]{2}{*}{ Consumo (kWh) } & \multicolumn{4}{|c|}{ Quantidade de aparelhos de ar condicionado } \\
\hline & & 0 & 1 & 2 & 3 \\
\hline \multirow[t]{2}{*}{ Área 1} & setembro & $327,6 a$ & $483,7 a$ & $449,0 a$ & - \\
\hline & março & $309,41 b$ & $566,27 a$ & $660,67 a$ & - \\
\hline \multirow[t]{2}{*}{ Área 2} & setembro & $469,6 b$ & $664,5 \mathrm{ab}$ & $891,8 a b$ & $1059,7 \mathrm{a}$ \\
\hline & março & $432,0 \mathrm{~b}$ & $577,3 \mathrm{ab}$ & $932,7 \mathrm{a}$ & 936,3а \\
\hline \multirow[t]{2}{*}{ Área 3} & setembro & $588,6 b$ & $929,2 \mathrm{ab}$ & - & $1402,3 a$ \\
\hline & março & $542,5 b$ & $915,2 \mathrm{ab}$ & - & $1345,3 a$ \\
\hline
\end{tabular}

Nota: Médias seguidas de letras diferentes, na linha, diferem $(\mathrm{P}<0,05)$ pelo teste Tukey.

Tabela 4 - Frequência das residências que ligam ar condicionado apenas quando está muito calor (A), deixam sempre ligados (B) e ligam apenas durante a noite, nos dias quentes (C), nas áreas 1, 2 e 3, São Paulo/SP, 2007.

Table 4 - Frequency of residences that turn on the air conditioning only when it is too hot (A), leave it always on (B) and turn it on only at night, in hot days (C), in the areas 1, 2 and 3, São Paulo/SP, 2007.

\begin{tabular}{lcccc}
\hline & & Frequência & C & Total \\
\hline Área 1 & A & B & 3 & 6 \\
Área 2 & 2 & 1 & 4 & 7 \\
Área 3 & 2 & 0 & 7 & 8 \\
\hline
\end{tabular}

$\mathrm{P}=0,4009$.

\section{DISCUSSÃO}

Os resultados analisados através dos questionários mostraram a dificuldade de mensurar a influência de áreas mais vegetadas (refletida na menor necessidade de uso de aparelhos de ar condicionado) no valor final de consumo de energia elétrica, com base nos dados coletados. Além dos fatores físicos (tais como presença de equipamentos elétricos), têm-se também fatores subjetivos, tais como hábito de uso de aparelhos de refrigeração - variando de acordo com o gosto de cada indivíduo - não sendo possível mensurar, através das contas de energia, tal influência.

Tais resultados confirmam pesquisas na área que, mesmo com diferentes metodologias, apontam para uma grande dificuldade de obter, apenas por dados coletados, uma relação direta entre uso de aparelhos de refrigeração e presença de vegetação, principalmente, segundo McPherson; Rowntree e Wagar (1995), pela dificuldade de isolar o efeito da vegetação no valor final do consumo.

Em relação aos questionários, pode-se dizer que cabe, em pesquisas futuras, acrescentar outro tipo de abordagem, mais aprofundada em relação ao hábito de uso de eletrodomésticos nas residências, não só de aparelhos de ar condicionado como também de refrigeradores, os quais têm seu uso (e consumo) variando de acordo com a época do ano. Dessa forma, seria possível analisar a contribuição de cada um dos equipamentos no valor final do consumo de energia elétrica. Ademais, seria conveniente o monitoramento do consumo em cada aparelho, facilitando a obtenção de sua contribuição individual no consumo final. A prioridade atribuída aos dados reais coletados, por sua vez, é fundamentada no pressuposto de que as informações assim obtidas são mais confiáveis, podendo servir - com maior precisão - como base tanto para outras pesquisas, como para a confecção de modelos teóricos de simulação.

\section{CONCLUSÃO}

Conclui-se que o uso do questionário não foi eficiente para estabelecer uma relação entre a presença de vegetação viária, o consumo de energia elétrica em residências e a presença e o uso de aparelhos de refrigeração. Isso porque não houve diferença estatística tanto entre os hábitos de uso de aparelhos de ar condicionado, quanto na quantidade de tais aparelhos,

Revista Árvore, Viçosa-MG, v.35, n.3, Edição Especial, p.641-648, 2011 

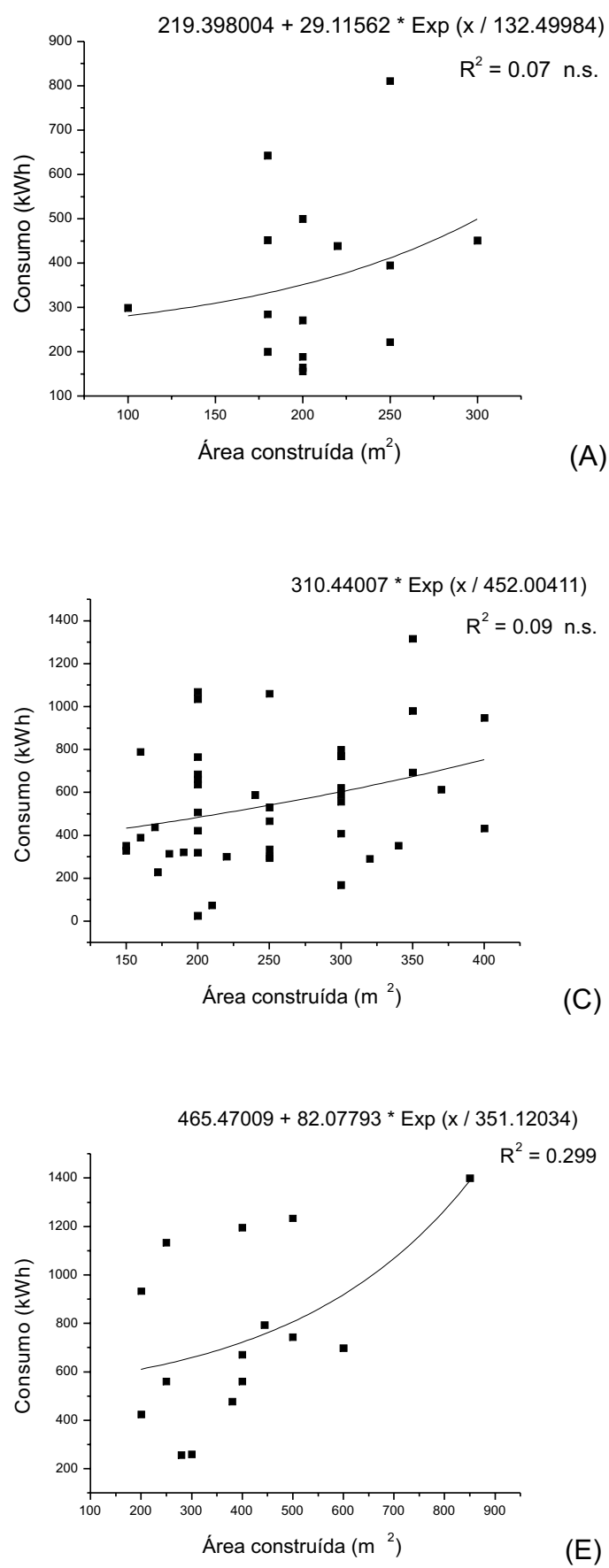
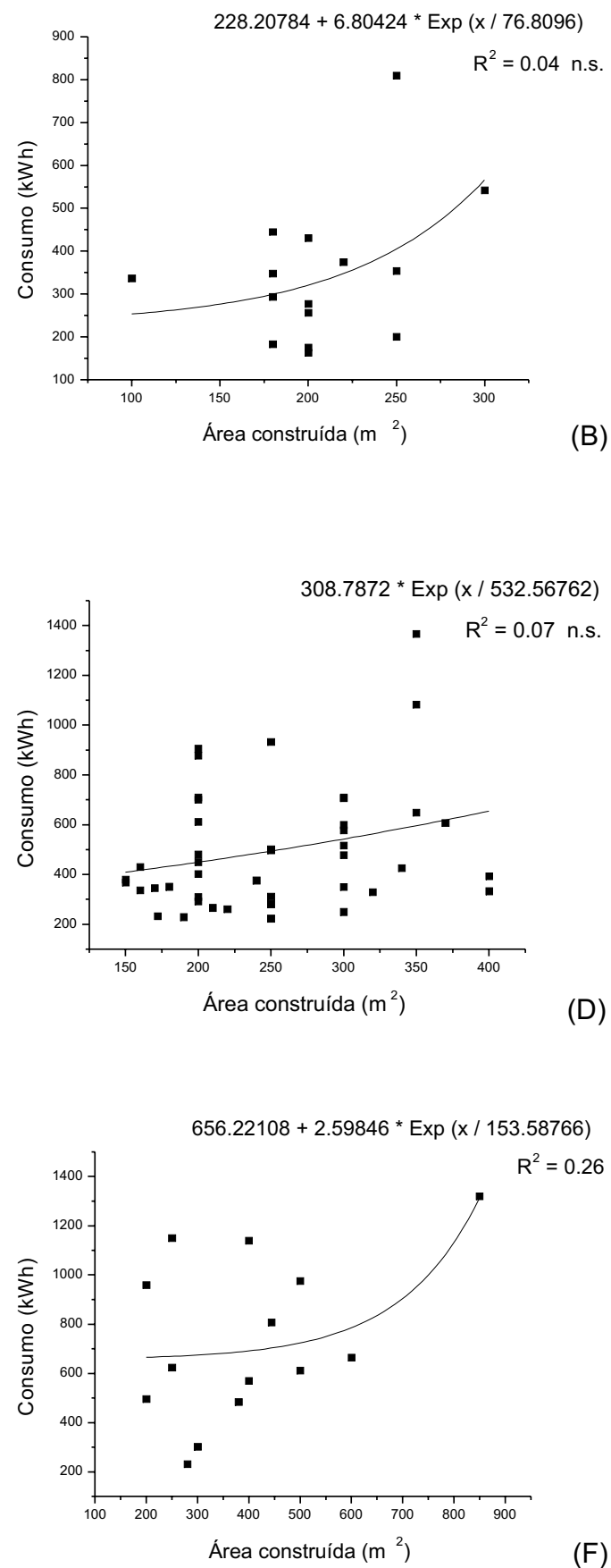

Nota: n.s. = não significativo.

Figura 2 - Regressão exponencial entre consumo (kWh) e área construída (m²): área 1 em setembro de 2006 (A) e março de 2007 (B); área 2 em setembro de 2006 (C) e março de 2007 (D); área 3 em setembro de 2006 (E) e março de 2007 (F).

Figure 2 - Exponential regression between consumption ( $k W h$ ) and built area (m2): area 1 in September 2006 (A) and March 2007 (B), Area 2 in September 2006 (C) and March 2007 (D) Area 3 in September 2006 (E) and March $2007(F)$. 
nas três áreas. Ao contrário, o consumo de energia elétrica diferiu entre as áreas, ou seja, apenas com os dados coletados nos questionários não foi possível isolar o efeito dos aparelhos de ar condicionado no valor total do consumo de energia elétrica, já que tal consumo variou nas três áreas, mas a quantidade e o uso não diferiram.

\section{AGRADECIMENTOS}

À FAPESP - Fundação de Amparo à Pesquisa do Estado de São Paulo, pela concessão de bolsa de estudos que possibilitou a execução desta pesquisa.

\section{REFERÊNCIAS}

BRETZ, S.; AKBARI, H.; ROSENFELD, A. Practical issues for using solar-reflective materials to mitigate urban heat islands. Atmospheric Environment, v.32, n.1, p.95-101, 1998.

CAVALCANTI, R. C. O consumo energético residencial em Campo Grande e a eficiência energética. 2002. 123f. Dissertação (Mestrado em Engenharia Civil) - Universidade Federal de Santa Catarina, Florianópolis, 2002.

ESTADOS UNIDOS. Departament of Energy. Disponível em: <http://www.eren.doe.gov>. Acesso em: 28 jun. 2007.

FRANCISCO, E. R. Relação entre o consumo de energia elétrica, a renda e a caracterização econômica de famílias de baixa renda do município de São Paulo. 2006. 349f. Dissertação (Mestrado em Administração de Empresas) Escola de Administração de Empresas de São Paulo, Fundação Getulio Vargas, São Paulo, 2006.

GOLDEMBERG, J.; VILLANUEVA, L. D.

Energia, meio ambiente \&

desenvolvimento. 2.ed. São Paulo: EDUSP, 2003. 226p.

HILDEBRAND, E.; GRAÇA, L. R.; MILANO, M. S. Distância de deslocamento dos visitantes dos parques urbanos em Curitiba-PR. Floresta e Ambiente, v.8, n.1, p.76-83, 2001.

KEEBLE, E. J.; COLLINS, M.; RYSER, J. The potential of land-use planning and development control to help achieve favorable microclimates around buildings: a European review. Energy and Buildings, v.15/16, p.823-836, 1990/91.
McPHERSON, E. G.; ROWNTREE, R.; WAGAR, A. Energy-efficient landscapes. In: BRADLEY, G. A. (Ed.). Urban forest landscapes: integrating multidisciplinary perspectives. Seattle: University of Washington Press, 1995. p.150-163.

PORFÍRIO, T. H. C. et al. Formas de interação de três bairros periféricos com o Parque Municipal das Mangabeiras, Belo Horizonte, Minas Gerais. Revista Árvore, v.30, n.6, p.1030-1038, 2006.

ROSENFELD, A. H et al. Cool communities: strategies for heat island mitigation and smog reduction. Energy and Buildings, v.28, n.1, p.51-62, 1998.

SANTAMOURIS, M. On the built environment the urban influence. In: SANTAMOURIS, M. Energy and climate in the urban built environment. Greece: James \& James, 2001. p.3-18.

SCHILLER, S.; EVANS, J. M. Training architects and planners to designd with urban microclimates. Atmospheric Environment, v.30, n.3, p.449-454, 1996.

SPIRN, A. W. O jardim de granito. São Paulo: EDUSP, 1995. 345p.

VELASCO, G. D. N. et al. Aplicação do índice de vegetação NDVI (Normalized Difference Vegetation Index) em imagens de alta resolução no município de São Paulo e suas limitações. Revista da Sociedade Brasileira de Arborização Urbana, v.2, n.3, p.1-12, 2007.

VIEIRA, C. H. S. D.; BIONDI, D. Análise da dinâmica da cobertura vegetal de Curitiba, PR (de 1986 a 2004), utilizando imagens Landsat TM. Revista Árvore, v.32, n.3, p.479-487, 2008.

WILMERS, F. Green for melioration of urban climate. Energy and Buildings, v.11, p.289-299, 1988.

YUAN, F.; BAUER, M. E. Comparison of impervious surface area and normalized difference vegetation index as indicators of surface urban heat island effects in Landsat imagery. Remote Sensing of

Environment, v.106, p.375-386, 2007. 